\title{
PolLux: A new facility for soft x-ray spectromicroscopy at the Swiss Light Source
}

\author{
J. Raabe, ${ }^{1, a)}$ G. Tzvetkov, ${ }^{2}$ U. Flechsig, ${ }^{1}$ M. Böge, ${ }^{1}$ A. Jaggi, ${ }^{1}$ B. Sarafimov, ${ }^{1}$ \\ M. G. C. Vernooij, ${ }^{3}$ T. Huthwelker, ${ }^{1}$ H. Ade, ${ }^{4}$ D. Kilcoyne, ${ }^{5}$ T. Tyliszczak, ${ }^{5}$ R. H. Fink, ${ }^{2}$ and \\ C. Quitmann ${ }^{1}$ \\ ${ }^{1}$ Swiss Light Source, Paul Scherrer Institut, 5232 Villigen-PSI, Switzerland \\ ${ }^{2}$ Physikalische Chemie II and ICMM, Universität Erlangen-Nürnberg, 91058 Erlangen, Germany \\ ${ }^{3}$ Eidgenössische Materialprüfungsanstalt (EMPA), 8600 Dübendorf, Switzerland \\ ${ }^{4}$ Department of Physics, North Carolina State University, Raleigh, North Carolina 27695-8202, USA \\ ${ }^{5}$ Advanced Light Source, Lawrence Berkeley National Laboratory, Berkeley, California 94720, USA
}

(Received 6 May 2008; accepted 21 October 2008; published online 13 November 2008)

\begin{abstract}
We report on the successful installation and operation of a scanning transmission x-ray microspectroscope (STXM) at the PolLux facility at the Swiss Light Source. This integration of an advanced STXM with improved sample handling capabilities and a novel beamline provides unique capabilities. PolLux uses linearly or circularly polarized x-rays from a bending magnet with an extended photon energy range $(200-1400 \mathrm{eV})$. It is therefore well suited to determine a sample's quantitative chemical composition, molecular orientation, or thickness of organic as well as condensed matter materials. The local magnetic state of magnetic thin films is accessible through fast helicity switching by steering the electron beam off axis through the bending magnet. Ex vacuo girder movers allow fast and highly reproducible $(<1 \mu \mathrm{m})$ alignment of the instrument with respect to the photon beam. The present spatial resolution is $\sim 20 \mathrm{~nm}$, limited by the zone plates utilized. The instrument has the stability and positional resolution to operate with much higher resolution optics as it becomes available. In addition to characterization experiments, we present several typical examples from materials research and environmental science to exemplify the capabilities. () 2008 American Institute of Physics. [DOI: 10.1063/1.3021472]
\end{abstract}

\section{INTRODUCTION}

The recent advances in nanotechnology have led to the development of new experimental techniques to investigate nanoscale materials with unprecedented resolution. The various scanning probe techniques [e.g., scanning tunneling microscopy or atomic force microscopy (AFM) and their related techniques] have revolutionized modern surface and interface science allowing atomic resolution. Most of the investigations with AFM often rely on the correlation with morphology. Electron microscopy can compete in terms of spatial resolution with the above mentioned scanning probe techniques and in the case of transmission electron microscopy (TEM) is not limited to morphology information. Highresolution TEM determines atomic positions in bulk with highest accuracy and full atomic resolution, i.e., it yields bulk information at ultimate spatial resolution.

However, obtaining the morphological information is often insufficient and the electronic properties, element composition, chemical state, and - in some cases-the magnetic properties of nanoscale materials have to be explored. This requires the combination of high spatial resolution with detailed spectroscopic information. Based on their unique interaction with matter, soft $\mathrm{x}$-rays have developed into a powerful tool to gain insight into the electronic properties and

\footnotetext{
${ }^{a)}$ Author to whom correspondence should be addressed. FAX: +41-56-310
} 3151. Tel.: +41-56-310-5193. Electronic mail: joerg raabe@psi.ch. chemical or magnetic state in condensed matter. ${ }^{1}$ Usually the characteristic properties vary across the sample and the spatial distribution of these variations has to be investigated at sufficiently high spatial resolution. Soft x-ray absorption spectroscopy combined with high spatial resolution is an ideal tool for such tasks, since it provides the necessary information with high sensitivity, minimum sample preparation, and flexibility of using a broad range of environments, such as magnetic fields, liquids, and gas flow. In particular, the near-edge $\mathrm{x}$-ray absorption fine structure (NEXAFS) shows superior sensitivity in organic materials, ${ }^{2}$ since the excitations of $1 s$ electrons of the light elements carbon, nitrogen, and oxygen (i.e., the major constituents in organic molecules) into unoccupied molecular orbitals/electronic states are governed by the local chemical surrounding of the excited atom.

In order to achieve high spatial resolution in scanning transmission soft $\mathrm{x}$-ray microspectroscopy, Fresnel zone plates (FZPs) are commonly utilized to focus the x-rays to a point focus. Images are formed from raster scanning the sample through the focal point while measuring the transmitted intensity $I_{\text {trans }}$ using an x-ray detector. By varying the photon energy $E$ and the polarization $\vec{P}$ or sample parameters such as the temperature, a multidimensional data set $I_{\text {trans }}(x, y, E, \vec{P}, \ldots)$ is obtained. By comparing the data to the well-known absorption edges of the elements ${ }^{3}$ and to reference samples, information about the elemental composition, 
chemical bonding, sample morphology, and magnetic order can be obtained. Since the pioneering work of Kirz and Rarback $^{4}$ several such scanning transmission $\mathrm{x}$-ray microscope (STXM) instruments have been built ${ }^{5-11}$ and have thus become important tools for micro- and nanocharacterization in material sciences and condensed matter research.

Similar microspectroscopic experiments are also available in so-called full-field TXMs, which reduce image acquisition times to seconds by transferring a magnified two-dimensional image onto a charge coupled device (CCD) camera. ${ }^{12,13}$ This requires a different microscope scheme using a condenser lens in front and a magnifying micro-FZP behind the sample. Spectroscopic imaging requires the use of an adequate monochromator as, e.g., presently used at BESSY (U41 beamline). ${ }^{14}$

The present paper describes the features of the present PolLux-STXM, which is installed at a bending magnet of the $2.4 \mathrm{GeV}$ electron storage ring of the Swiss Light Source (SLS) (Villigen, Switzerland). The PolLux-STXM is based on the STXM installed at BL 5.3.2 of the Advanced Light Source (ALS) (Berkeley, USA) but has several significant improvements. It operates at an increased photon energy range from 200 to $1400 \mathrm{eV}$, since a high-energy grating has been implemented to the beamline, thus offering improved conditions to investigate, e.g., transition metal samples. The SLS top-up mode gives much higher stability to the electron beam allowing much higher integral count rates. In addition, the superior beam stability at the SLS and the implemented feedback systems even allows one to control the local tilt of the storage ring orbit (see Sec. II C). This results in circularly polarized light which at present can be switched from left- to right-handed circularly polarized light with repetition rates of $1 \mathrm{~Hz}$ at present $(10 \mathrm{~Hz}$ in near future) without moving any of the position sensitive mirrors of the monochromator. This operation mode will allow new experiments to explore magnetization dynamics in nanomagnetic materials based on the $\mathrm{x}$-ray magnetic circular dichroism contrast. Further improvements include, e.g., ex vacuo girder movers to easily align the instrument with respect to the illuminating beam. The successful installation of prototype STXM at the PolLux facility has triggered the commercial availability of STXMs to become a widely distributed nanoanalysis tool in modern synchrotron research.

In summary, the unique features of the PolLux facility are as follows.

- The alignment of the instrument with respect to the beamline is much easier, faster, and more flexible by the use of ex vacuo girder movers.

- The interferometer resolution is improved over prior instruments, allowing improved feedback and microscope stability.

- Due to the improved storage ring performance with respect to beam stability including fast-forward feedback implementation and the use of top-up injection at the SLS storage ring, PolLux offers new opportunities such as routine operation of fast helicity switching (presently $1 \mathrm{~Hz}$, in the future around $10 \mathrm{~Hz}$ or even faster) which is an important prerequisite in micromagnetic analysis (which in combina- tion with time-resolved studies will open new experimental analysis options). This is a novel feature, unique to the PolLux facility.

- The vacuum in the STXM chamber is approximately three orders of magnitude lower, thus enabling even secondary electron detection.

- In addition, PolLux covers a larger energy range than the ALS 5.3.2 STXM and thus allows the characterization of a much wider range of materials.

- Lastly, superior spatial resolution has been demonstrated. ${ }^{29}$

\section{INSTRUMENT COMPONENTS AND THEIR PERFORMANCE}

\section{A. Monochromator, PolLux beamline}

Similar to the original concept of Kirz and Rarback, ${ }^{4}$ the PolLux instrument uses a spherical grating monochromator with horizontal dispersion, a Fresnel zone plate (FZP), an order selecting aperture (OSA), and a raster scanned sample. ${ }^{7}$ The technical implementation is based on the design of the beamline 5.3.2 STXM at the ALS. ${ }^{5,16}$ The PolLux instrument was designed as a robust, user friendly experiment providing large flexibility for different sample environments.

The source is a bend magnet with a critical energy of 5.5 $\mathrm{keV}^{17}$ The optical principle of the beamline is shown in Fig. 1. A horizontally deflecting toroidal mirror creates a horizontal focus of the bending magnet source at the entrance slit (S1) with a magnification of $M_{\mathrm{hor}}=1: 1$. The monochromator with two spherical gratings (300 and 600 lines $/ \mathrm{mm}$, respectively) in a fixed $\theta$-mount disperses in the horizontal plane. In the vertical direction the source is imaged by the toroidal mirror $M_{\text {vert }}=1.8: 1$ onto the exit slit (S2) that is acting as secondary source for the FZP located $1 \mathrm{~m}$ downstream. The details of the design and performance predictions are provided in Ref. 18.

The principle of the optical design follows a concept developed at the ALS, ${ }^{16,19}$ which demonstrated the possibility to build a competitive STXM instrument at a bending magnet without the complexity and costs of an insertion device beamline. For maximum spatial resolution, the exit slit must limit the illumination of the zone plate to the coherent fraction of the incident photon phase space. ${ }^{20,21}$ This leads to the design of a long beamline with small magnifications. The fraction of the phase space of the source which cannot be coherently imaged by the zone plate is used to deliberately overfill the slits. This improves the signal-to-noise ratio because it reduces the sensitivity to drifts and vibrations. The entrance slit (S1) can be used to gain photon flux at the expense of energy resolution without sacrificing the spatial resolution, which is only determined by the size of the exit slit (S2) and the properties of the zone plate.

Compared to the ALS beamline 5.3.2, PolLux covers a wider photon energy range that extends to $E=1400 \mathrm{eV}$. This allows investigations on correlated electron systems $(\mathrm{Cu} L$ edge), GaAs and its variants ( $\mathrm{Ga} L$ and As $L$ edges) and on rare-earth containing materials ( $M_{\mathrm{IV}-\mathrm{V}}$ edges up to holmium). The configuration of PolLux also offers easy access to circularly polarized light in addition to the standard linear polar- 


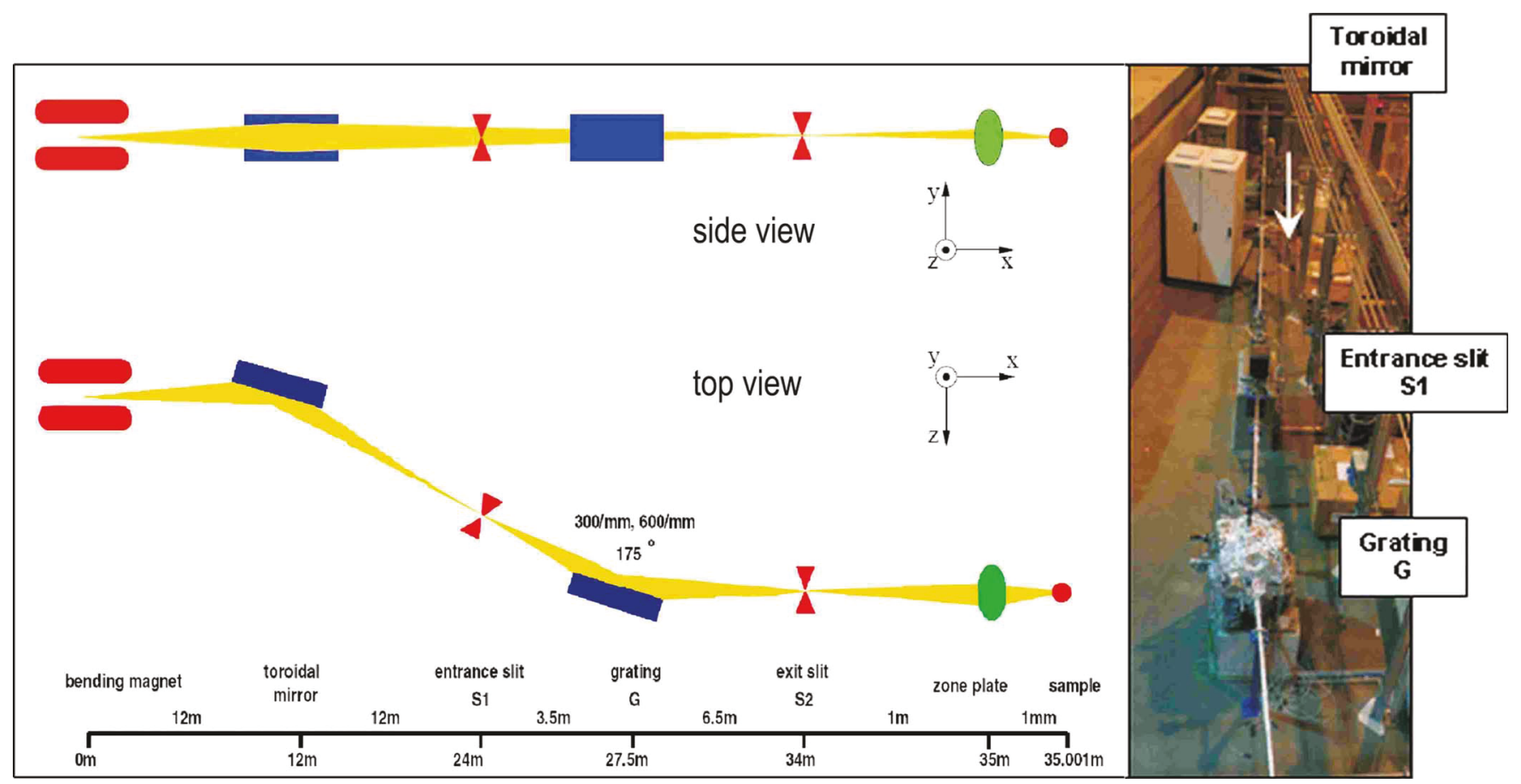

FIG. 1. (Color online) Optical layout of the PolLux beamline (not to scale) showing the bending magnet source followed by the toroidal mirror and the spherical grating monochromator. These create a secondary source at the exit slit (S2) illuminating the FZP which produces the focal spot across which the sample is scanned. The photograph on the right shows several of the beamline components.

ization, enabling the investigation of magnetic materials. Circularly polarized light is obtained by a controlled local tilt of the storage ring orbit, a new and unique feature which will be described in Sec. II C.

In the PolLux monochromator, the gratings $G$ rotate around the vertical $y$-axis formed by two flexure hinges. The two gratings are mounted on a single carriage allowing a vertical translation guided by cross-roller bearing stages. They can be individually adjusted in four degrees of freedom $\left(\mathrm{z}, R_{x}, R_{y}, R_{z}\right)$ allowing an alignment of the grating surface and grove orientation with respect to the rotation axis of the monochromator. The grating rotation $R_{y}$ and translation $y$ are remotely controlled by motors and encoders located outside the vacuum. Details of the mechanical design and characterization are published by Henein et al. ${ }^{22}$

The vacuum separation between the STXM and the beamline is achieved by a silicon nitride membrane $\left(\mathrm{Si}_{3} \mathrm{~N}_{4}\right.$, $100 \mathrm{~nm}$ thick) located just upstream of the FZP. Measurements with the STXM can then be either at atmospheric pressure (air or He) or in a vacuum of $\sim 10^{-6}$ mbar (which is more than three orders of magnitude smaller than for the ALS STXMs). A gas cell, located between the exit slit S2 and the $\mathrm{Si}_{3} \mathrm{~N}_{4}$ membrane, is used for absolute calibration of the photon energy and for energy resolution measurements. For this purpose a $100 \mathrm{~nm}$ thick $\mathrm{Al}$ foil mounted in a manual gate valve can be inserted, which then separates the gas in the cell from the beamline vacuum.

\section{B. Monochromator commissioning results}

A number of photoion yield measurements at different absorption edges have been carried out using the gas cell in order to characterize the energy resolution of the beamline.
The well known $\mathrm{N}_{2} 1 s \rightarrow \pi^{*}$ transition at about $400 \mathrm{eV}$ was used to optimize the energy resolution using the 300 lines/mm grating (G1). An example is shown in Fig. 2, using equal slit widths of $10 \mu \mathrm{m}$ for S1 and S2. Because of the intrinsic linewidth of the $\mathrm{N}_{2} 1 s \rightarrow \pi^{*}$ transition, the energy resolution is not simply given by the width of the peaks but must be estimated by the ratio of the third maximum to the first minimum. ${ }^{23}$ As seen in Fig. 2, this ratio is 0.8 , indicating a resolving power above 5000, which is adequate for the NEXAFS spectroscopy experiments performed at PolLux.

The relation between energy resolution and photon flux,

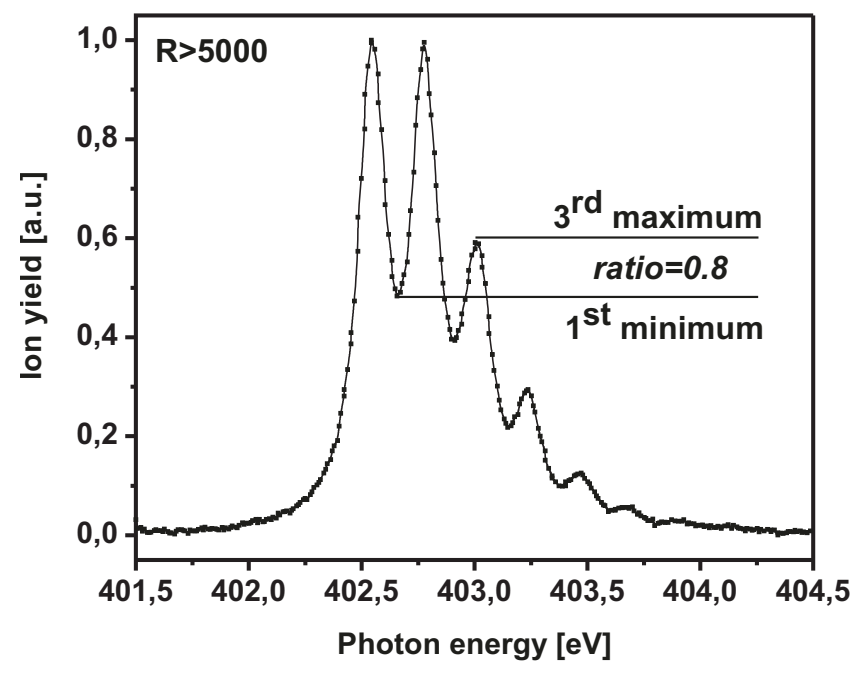

FIG. 2. Measured photoion yield at the nitrogen $1 s \rightarrow \pi^{*}$ transition using the gas cell located between exit slit and FZP of the PolLux beamline (300 lines $/ \mathrm{mm}$ grating, $10 \mu \mathrm{m}$ slits). The intensity ratio of the first minimum to the third maximum (0.8) indicates an energy resolution in excess of $E / \Delta E$ $\sim 5000$ (Ref. 23). 


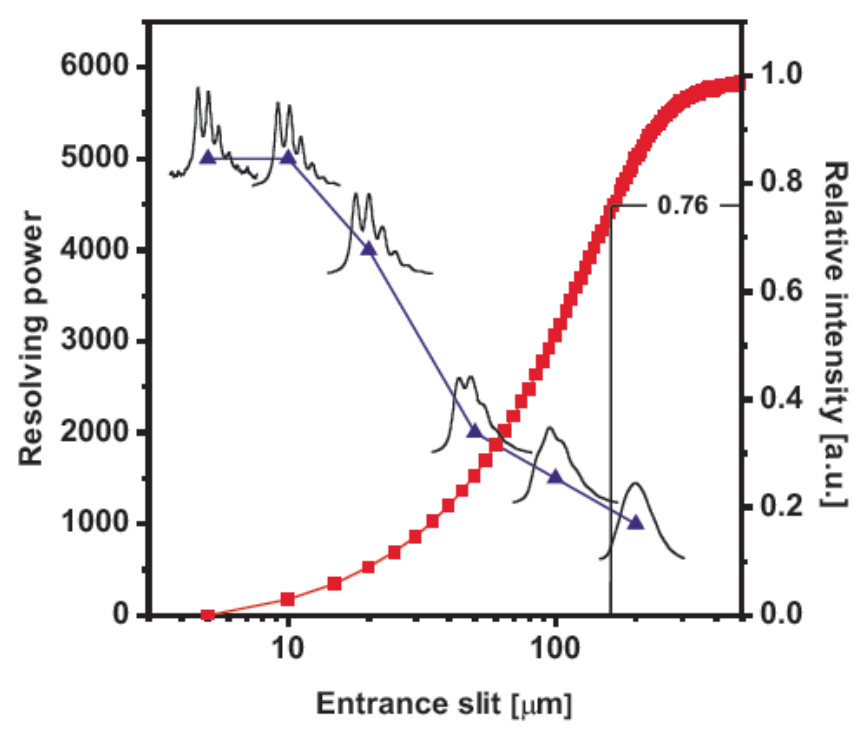

FIG. 3. (Color online) Resolving power (left scale) and relative intensity (right scale) as function of the entrance slit width measured with the 300 lines/mm grating at an exit slit of $50 \times 50 \mu \mathrm{m}^{2}$. The resolving power has been determined from the $\mathrm{N}_{2}$ spectra shown as insets. The lines indicate the resolving power for equal entrance and exit slits matched to the horizontal focus width at the entrance slit (FWHM $=165 \mu \mathrm{m})$.

which is of interest for practical experiments, is shown in Fig. 3 for the 300 lines/mm grating. The optical design allows a trade-off between photon flux and resolution by the settings of the exit slit opening (S2) and entrance slit (S1). As illustrated in Fig. 3, the energy resolution can be degraded from a value of $\Delta E / E=5000$ to a value of $\Delta E / E$ $=2000$ by opening the entrance slit $\mathrm{S} 1$ (parallel to the energy dispersion plane) from 20 to $50 \mu \mathrm{m}$ while maintaining the exit slit (S2) at a nominal $50 \times 50 \mu \mathrm{m}^{2}$. This is accompanied by an increase in the photon flux by a factor of 2.5 as shown to the right in Fig. 3. Assuming a Gaussian intensity distribution at S1 we obtain the full width at half maximum (FWHM) horizontal focus at the entrance slit from the $76 \%$ value of the relative intensity. The experimental value of $165 \mu \mathrm{m}$ is in a good agreement with the value of $146 \mu \mathrm{m}$ obtained from ray tracing.

The resolving power has been checked at other photon energies using other gases (Ar: $240 \mathrm{eV} ; \mathrm{CO}_{2}: 280$ and 540 $\mathrm{eV} ; \mathrm{N}_{2}: 400 \mathrm{eV} ; \mathrm{Ne}: 870 \mathrm{eV}$ ). The results are shown at the very low energy end of the instrument for the $\operatorname{Ar} L_{2,3}$ absorption edge near $240 \mathrm{eV}$ using the 300 lines/mm grating [Fig. 4(a)] and for the Ne 1s-np absorption edge near $867 \mathrm{eV}$ using the 600 lines $/ \mathrm{mm}$ grating [Fig. 4(b)].

The photon flux on the sample is shown in Fig. 5 for typical experimental conditions. To correctly measure it we include the absorption by the $\mathrm{Si}_{3} \mathrm{~N}_{4}$ vacuum window, the zone plate, and the order selecting aperture. In addition, we keep the spectral resolution constant by varying the width of the entrance slit (S1), and adjust for the energy dependant focal length of the FZP. ${ }^{24}$ The photon flux at the sample was measured using the silicon photodiode which is one of the two standard detectors in the instrument.

The absorption edges of carbon $(280 \mathrm{eV})$, nitrogen $(400$ $\mathrm{eV})$, and oxygen $(540 \mathrm{eV})$ result in local minima in the measurement because of contamination on the optical elements
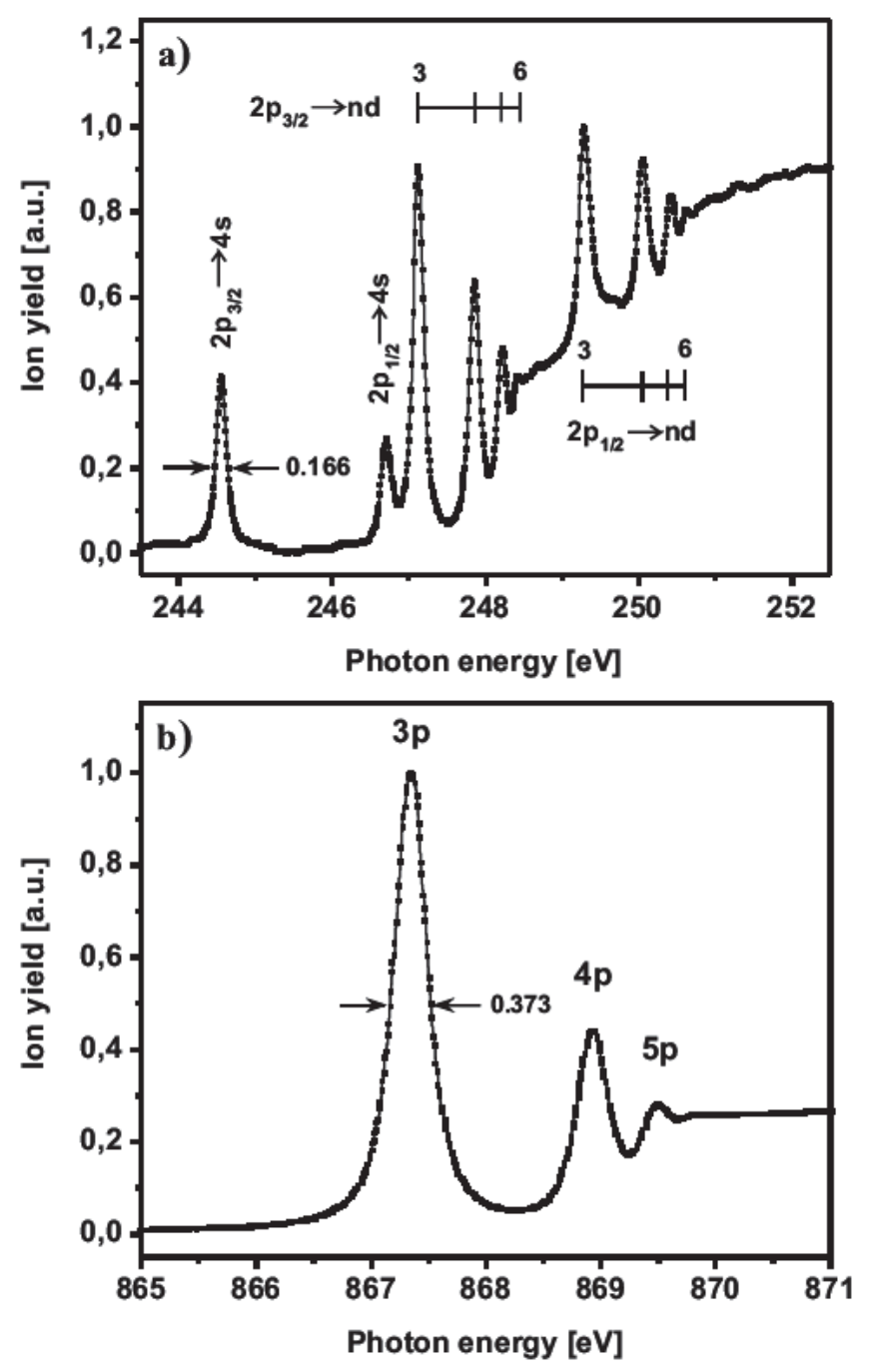

FIG. 4. (a) Argon $L_{2,3}$ photoion yield measurement for 300 lines $/ \mathrm{mm}$ grating and $10 \mu \mathrm{m}$ exit slit setting. (b) Measured $1 s-n p$ transition series of neon with 600 lines/mm grating.

and absorption in the $\mathrm{Si}_{3} \mathrm{~N}_{4}$ membrane. The small dip at 265 $\mathrm{eV}$ is a result of light absorbed by oxygen contamination due to second order contributions from the monochromator. Below $200 \mathrm{eV}$, calculations predict a high contribution from third order light, ${ }^{18}$ which is confirmed by the measurement. This limits the useful range of the PolLux experiment toward low photon energies. The region beyond $1000 \mathrm{eV}$ still provides reasonable flux that can be exploited for experiments.

\section{Polarization switching for $x$-ray magnetic circular dichroism analysis}

Circularly polarized light can be generated by a localized angular tilt of the electron beam orbit within the dipole magnet. ${ }^{15}$ Here, we introduce this tilt by means of a vertical asymmetric bump produced by four successive dipole correctors which are part of the standard SLS orbit correction system. ${ }^{25}$ The maximum tilt amplitude is $\Delta \psi= \pm 300 \mu \mathrm{rad}$. To compensate for the increase in the betatron coupling, two skew quadrupoles, up- and downstream, of the PolLux dipole magnet are used. Presently, the switching is done quasistatically $(\sim 1 \mathrm{~s}$ switching time). Fast switching with an expected upper limit of about $10 \mathrm{~Hz}$ is presently being 

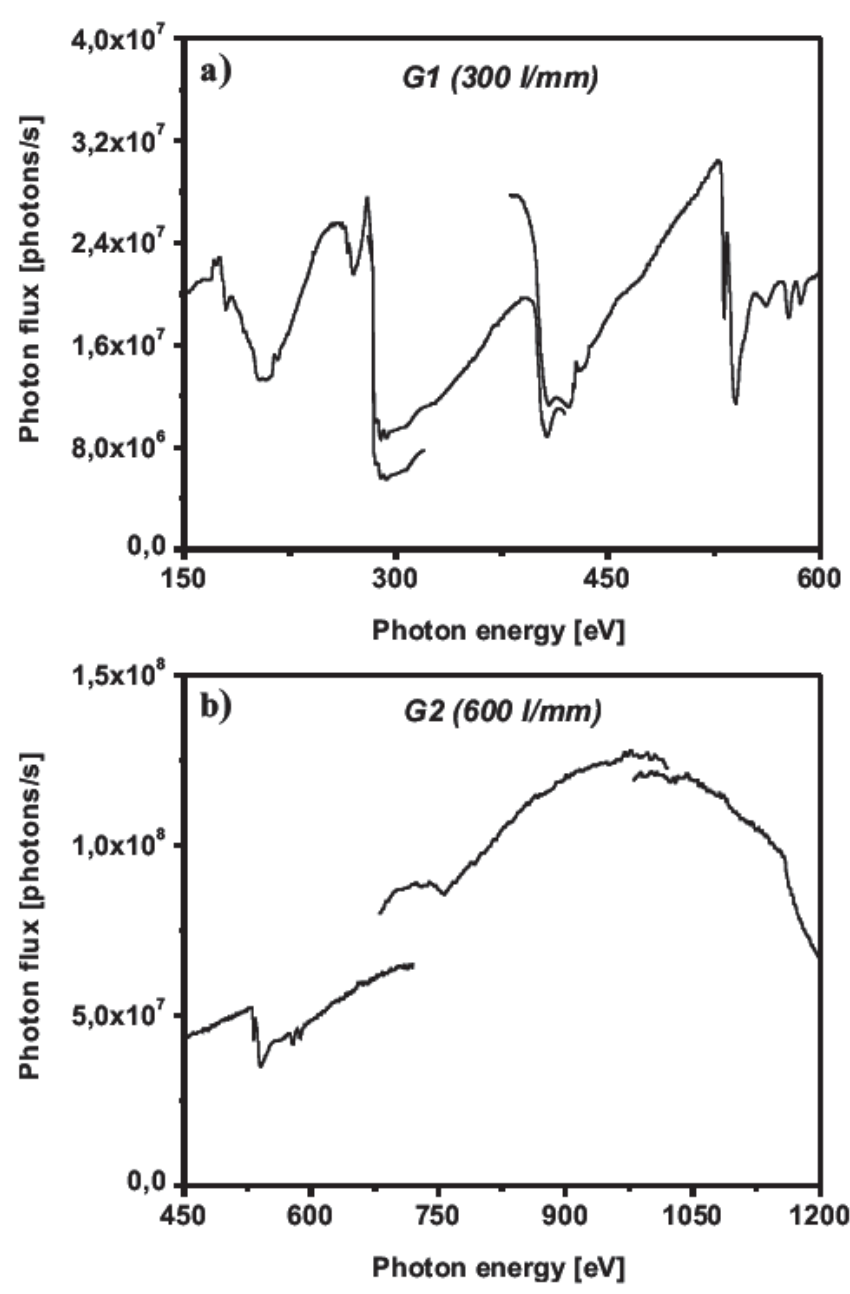

FIG. 5. Measured photon flux at the sample for the (a) 300 lines $/ \mathrm{mm}$ grating and (b) 600 lines/mm grating. The entrance slit width has been continuously adjusted with energy to keep the spectral resolving power constant at $E / \Delta E=2000$. The exit slit has been kept constant at $40 \times 40 \mu \mathrm{m}^{2}$. See text for details.

implemented. The orbit bumps are invisible to other beamlines because any orbit deviations are compensated by the fast-orbit feedback with an accuracy of a few micrometers and up to a cutoff frequency of $100 \mathrm{~Hz}$.

The principle of polarization switching is sketched in Fig. 6, where the upper panel shows the situation with $\Delta \Psi$ $=0$ which provides linear horizontal polarization. The opening angle of the dipole radiation is $\psi_{0}$ and the beamline vertical acceptance is $\psi_{A}$, which is given by the opening of the entrance slit. The lower panel shows the beam tilted downward by $\Delta \psi$, resulting in left circularly polarized light entering the beamline. For the STXM beamline, the usable vertical acceptance angle $\psi_{A}$ is limited by the vertical acceptance angle of the zone plate and can be calculated by

$$
\Psi_{A}=\frac{d_{\mathrm{FZP}}}{\ell_{\mathrm{FZP}}} \frac{1}{M_{\text {vert }}} \sim 430 \mu \mathrm{rad},
$$

where $d_{\mathrm{FZP}}=240 \mu \mathrm{m}$ is the zone plate diameter, $\ell_{\mathrm{FZP}}$ $=1000 \mathrm{~mm}$ is the distance between exit slit S2 and zone plate and $M_{\text {vert }}=1.8: 1$ is the vertical magnification of the
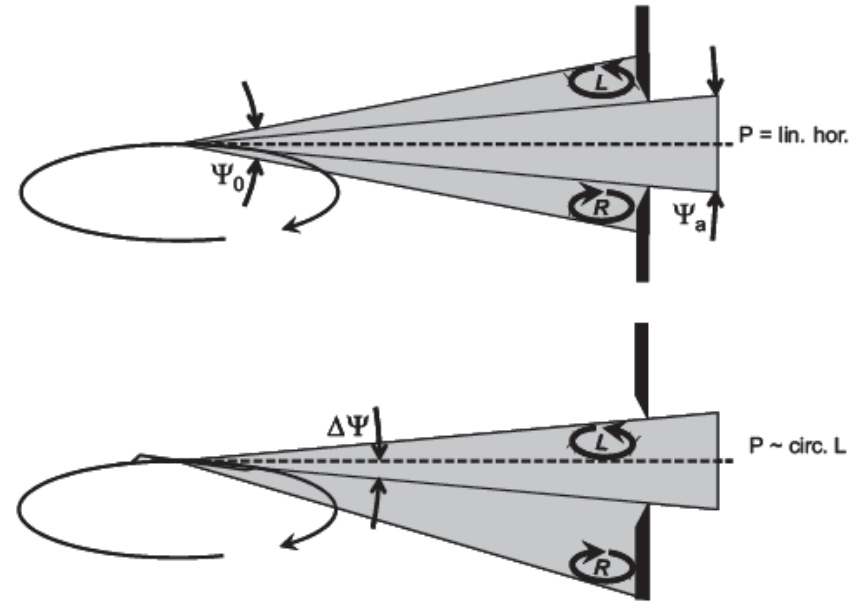

FIG. 6. Scheme showing how circularly polarized light is obtained from a bending magnet by tilting the storage ring orbit relative to the optical axis of the beamline. The beamline acceptance is $\psi_{A}$; the tilt angle of the orbit is $\Delta \psi$ $(\leq \pm 300 \mu \mathrm{rad})$.

first mirror. First results of magnetic imaging utilizing x-ray magnetic circular dichroism (XMCD) are presented in Sec. III A.

\section{PolLux end station}

The PolLux-STXM was developed by a collaboration of the ALS, North Carolina State University, the University of Erlangen-Nürnberg, the Paul Scherrer Institut, and the company ACCEL (Bergisch-Gladbach, Germany). ${ }^{26}$ The principle of the instrument is shown in Fig. 7. The FZP focuses the soft x-ray light to a diffraction limited spot. Higher diffraction orders are filtered out using an OSA, located between the FZP and the sample. The sample consists of a semitransparent silicon nitride membrane ${ }^{27}$ or a TEM grid on which the sample is mounted. The intensity of the transmitted $\mathrm{x}$-ray beam is measured by a large $(\varnothing \sim 0.5 \mathrm{~mm})$ area detector behind the sample. At present two types of detectors are available, a photodiode and a photomultiplier tube coupled to a scintillator (phosphor screen), which converts

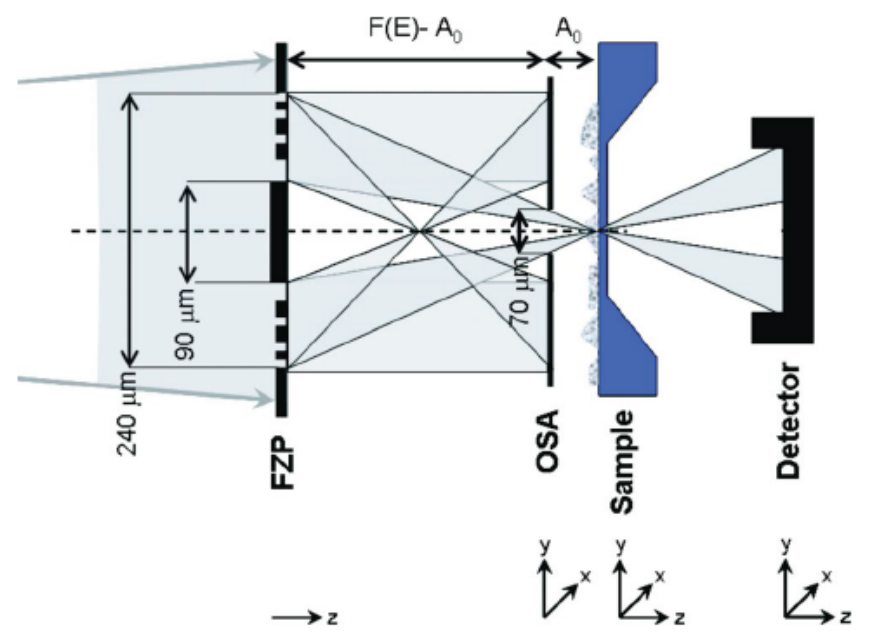

FIG. 7. (Color online) Scheme of a STXM showing the divergent beam downstream of the exit slit, FZP producing a diffraction limited focus, OSA selecting only the first-order focus, the sample, and the detector measuring the transmitted intensity. 
the x-rays to visible light. The photon energy dependent focal length of a zone plate $f(E)$ is given by $f(E)$ $=d_{\mathrm{FZP}} \Delta r(1 / c h) E$. For typical zone plate parameters (outer diameter $d_{\mathrm{FZP}}=240 \mu \mathrm{m}$, thinnest ring $\Delta r=35 \mu \mathrm{nm}$ ) this corresponds to an energy dependent focal length of $f(E)=a_{1} E$ $=7(\mu \mathrm{m} / \mathrm{eV}) E$. The OSA is positioned so that it provides maximum rejection of higher diffraction orders (both from the monochromator and the FZP) and maximum transmission of first-order light through its aperture of $70 \mu \mathrm{m}$. The distance between OSA and the sample $A_{0}$ is then typically $A_{0}=450 \mu \mathrm{m}$ at the $\mathrm{C} 1 \mathrm{~s}$ edge $(E=285 \mathrm{eV})$ and $800 \mu \mathrm{m}$ at the Fe $2 p$ edge $(E=709 \mathrm{eV})$. The motorized degrees of freedom for the different components are shown in Fig. 7. Imaging of samples mounted on the piezonanoscanner stage is accomplished by raster scanning $(x, y, z=$ const $)$ across the $\mathrm{X}$-ray focus. For spectroscopy, the FZP is moved along the $z$-direction (optical path) to track the energy dependence of the focal length, while the sample position is kept fixed. To achieve the required spatial resolution, tight control of the relative position of FZP and sample is necessary. It is realized by closed loop optical interferometry. ${ }^{5,28}$ The system used for PolLux has increased resolution over the 5.3.2. STXM (Agilent N1231A together with DeltaTau PMAC PCI lite motion controller) and offers therefore improved performance. The quality of the mechanics and the control are demonstrated by the smallest features that have been resolved. These are $15 \mathrm{~nm}$ walls separating $15 \mathrm{~nm}$ trenches in a FZP used as test object which was imaged using a FZP with $\Delta r=20 \mathrm{~nm}$ outmost zone width. ${ }^{29}$

To allow easy and reproducible positioning of the FZP with respect to the stationary X-ray beam, the entire STXM is supported by a five-axis alignment system mounted outside of the vacuum chamber. This "mover system" was originally designed for the girders of the SLS storage ring. ${ }^{30}$ It allows positioning along the $x$ and $y$ and rotation around the $x$-, $y$-, and $z$-axes with micrometer precision over a range of a few millimeters (for the rotation this corresponds to approximately microradiant precision and a milliradiant range). The precise alignment of the end station with respect to the photon source (illuminating beam) enables fast FZP exchange with a minimum of downtime ( $<15 \mathrm{~min})$.

The PolLux-STXM has been designed to allow operation in high vacuum. A turbomolecular pump, mechanically isolated by a bellow and mounted on an independent stand, allows pressures down to $10^{-6}$ mbar. The vacuum chamber provides easy access for sample exchange as well as the facility to provide fluids, gases, and cooling and for connecting electrical signals. It also provides ports for optical access, which will be used for optical levitation of individual nanoobjects. Extra ports may be utilized to implement a fast CCD camera thus offering simple implementation of differential phase contrast microscopy. Photographs of the PolLuxSTXM are shown in Fig. 8.

\section{RESEARCH EXAMPLES}

Soft x-ray STXM is an established technique for microspectroscopy of soft matter, ${ }^{2,31}$ magnetic materials, ${ }^{32,33}$ and environmental and geosciences ${ }^{34,35}$ as well as for bio-
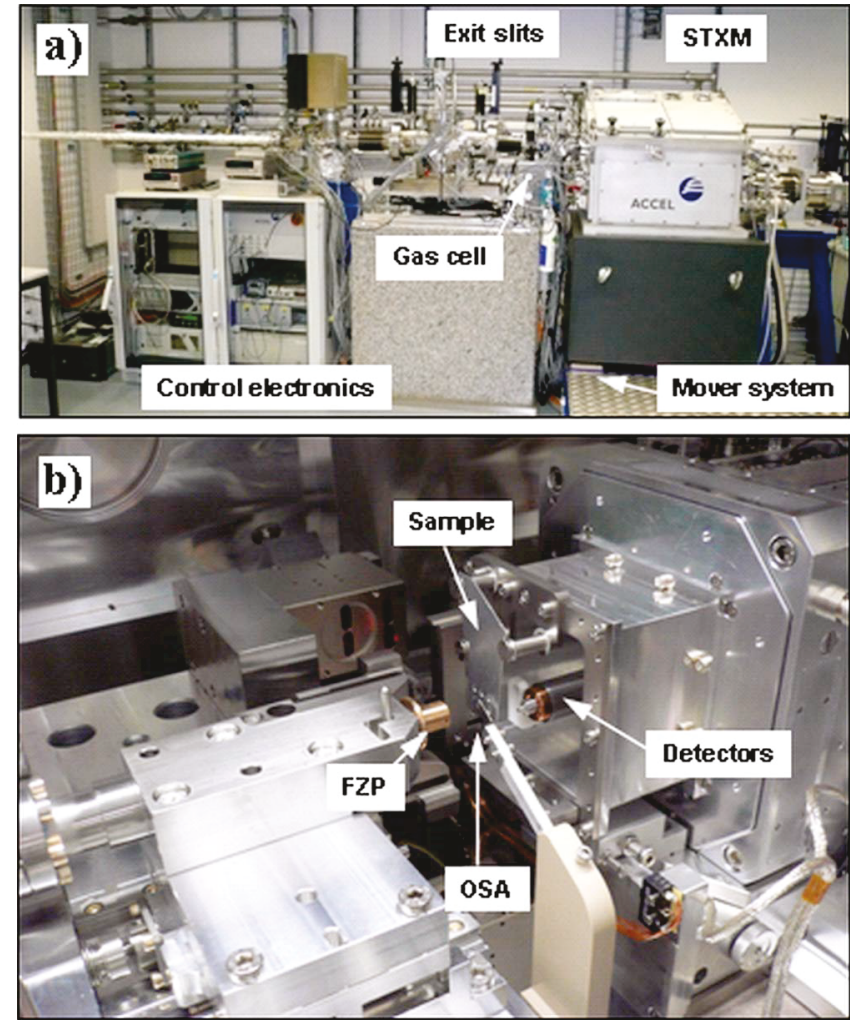

FIG. 8. (Color online) Photographs of the PolLux STXM: (a) inside the experimental hutch and (b) main elements of the setup.

logical imaging. ${ }^{36}$ With the increasing number of instruments available, more intense use is predicted and numerous new fields will make use of such type of microspectroscopy. Combining high spatial with high spectroscopic and even temporal resolution allows investigations of many properties relevant for micro- and nanostructured materials. The contrast in STXM is the spatially resolved x-ray transmission signal $I_{\text {trans }}$ which is given by $I_{\text {trans }}(\vec{r})=I_{0}$ $\times \exp \{-\mu(\vec{P}, E, Z) \cdot t(\vec{r})\}$, where $I_{0}$ and $I_{\text {trans }}(\vec{r})$ are the incident and transmitted x-ray intensities at position $\vec{r}$, respectively. The absorption coefficient $\mu$ depends on the polarization $\vec{P}$, the photon energy $E$, and the atomic number $Z$. The local thickness of the sample is $t(\vec{r})$. The sensitivity to the chemical elements comes primarily from the dependence of the absorption edges on the atomic number $Z$, but variations in the chemical state lead to significant changes in the position and intensity of absorption peaks in the near-edge region, thus providing detailed information on the chemical bonding. ${ }^{1,37}$ In the following, we show several examples illustrating the wide range of scientific questions that can be investigated using the PolLux beamline.

\section{A. Magnetic imaging and spectroscopy}

Since PolLux also provides circularly polarized light, it can be used for XMCD experiments. ${ }^{38}$ As an example, we show results on a CoPt multilayer sample. The sample consists of a multilayer structure of Co and Pt (total Co thickness $t=6 \mathrm{~nm}$ ) on top of a IrMn film which induces an exchange bias. It is known that CoPt forms wormlike domains magnetized perpendicular to the film plane. ${ }^{39}$ An image of 

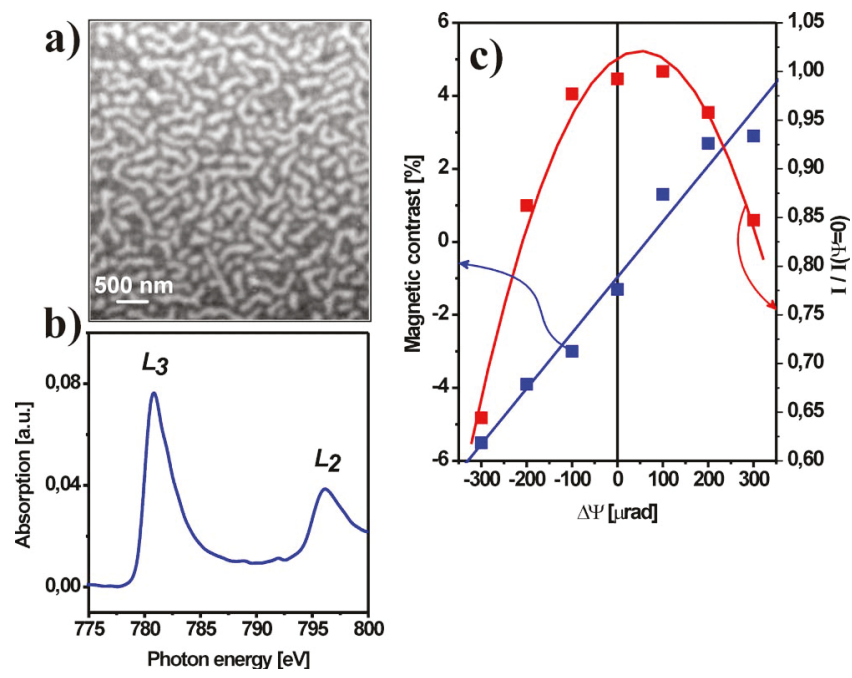

FIG. 9. (Color online) Magnetic imaging of a CoPt/IrMn multilayer sample (total Co thickness $=6 \mathrm{~nm}$ ). The well known worm domains of about 200 $\mathrm{nm}$ width are shown in (a), a spectrum taken at the Co $L$ edge in (b), and the magnetic contrast and relative intensity as a function of bump angle $\Delta \psi$ in (c).

these magnetic domains is shown in Fig. 9(a). They are visible as dark and bright regions of approximately $200 \mathrm{~nm}$ width in the dichroic transmission signal:

$$
I_{\mathrm{XMCD}}(\overrightarrow{\mathrm{r}})=\frac{[I(\overrightarrow{\mathrm{r}},+\Delta \Psi)-I(\overrightarrow{\mathrm{r}},-\Delta \Psi)]}{[I(\overrightarrow{\mathrm{r}},+\Delta \Psi)+I(\overrightarrow{\mathrm{r}},-\Delta \Psi)]} .
$$

The Co $L$-edge spectrum is shown in Fig. 9(b) for $C^{+}$helicity. To check the effect of the bump angle $\Delta \psi$ quantitatively, images were acquired as a function of $\Delta \psi$. The analysis is shown in Fig. 9(c). The magnetic contrast $\left(I_{\uparrow}-I_{\downarrow}\right) /\left(I_{\uparrow}+I_{\downarrow}\right)$ was calculated from the intensity on a domain magnetized up $\left(I_{\uparrow}\right)$ and from a domain magnetized down $\left(I_{\downarrow}\right)$. It increases linearly with the bump angle $\Delta \Psi$ at a rate of $0.15 \% / \mu \mathrm{rad}$. The relative intensity $I(\Delta \psi) / I(\Delta \psi=0)$ falls off quadratically, reflecting the beam profile folded with the acceptance of the beamline. At the maximum available tilt angle $\Delta \psi$ $=300 \mu \mathrm{rad}$ the magnetic contrast is about $4 \%$. When taking into account that the resonant absorption at the Co $L$ edge of this sample $\left(t_{\mathrm{Co}}=6 \mathrm{~nm}\right)$ is only about $20 \%$, this corresponds to about $20 \%$ effective dichroism in the Co signal. This value is surprisingly close to what can be obtained on beamlines using circularly polarized undulators, indicating a high degree of circular polarization. A setup to explore the magnetization dynamics with subnanosecond resolution is presently being developed.

\section{B. Spectromicroscopy of organic thin film}

During the past decade, scanning transmission x-ray spectromicroscopy has been extensively used to study a number of carbonaceous materials, such as polymers, biomolecules, and soil colloids. ${ }^{40-43}$ Since NEXAFS spectroscopy at the carbon $K$ edge is a measure of the geometric and electronic structure of the molecules using the characteristic C $1 s \rightarrow \pi^{*}$ and $\sigma^{*}$ resonances, STXM can fingerprint the chemistry of carbon containing samples. Major advantages

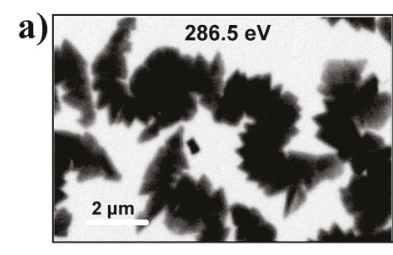

b)
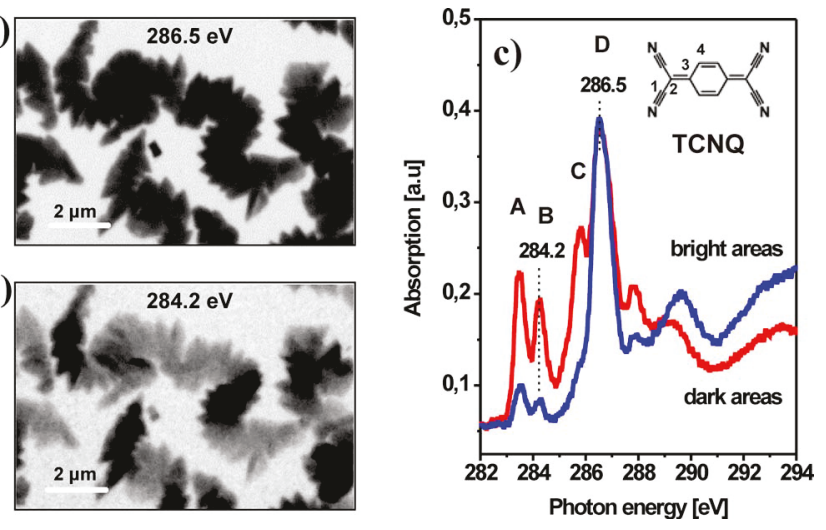

FIG. 10. (Color online) STXM transmission images at (a) $286.5 \mathrm{eV}$ and (b) $284.2 \mathrm{eV}$ of $200 \mathrm{~nm}$ thick TCNQ film grown on top of a $5 \mathrm{~nm}$ silver film; (c) $\mathrm{C} K$-edge NEXAFS spectra extracted from line scans taken across bright and dark microcrystallites shown in (b). The line scans were performed with $0.02 \mathrm{eV}$ energy step from 280 to $295 \mathrm{eV}$ with $40 \mathrm{~ms}$ dwell time per pixel.

of the technique include the possibility to perform quantitative compositional and orientational analyses of organic nanomaterials. ${ }^{2,44-47}$

In the following example we monitor the morphology and molecular orientation in ultrathin organic films. Fabrication of highly oriented and ordered films by controlled growth and the fundamental understanding of the interrelations between structural and electronic properties of such films are important for the future development of organic electronic devices. In this example, 7,7,8,8tetracyanoquinodimethane (TCNQ) films were grown on silver-covered $\mathrm{Si}_{3} \mathrm{~N}_{4}$ membranes by vacuum sublimation. TCNQ is a good electron acceptor and is known to form radical anion transfer complexes with a number of different metals. These complexes show a wide range of interesting electronic properties such as electrical conductivity and ferromagnetism.

Vacuum deposition of TCNQ on $5 \mathrm{~nm}$ thick silver films leads to the formation of s-shaped agglomerations of nanocrystals, which can be imaged using the PolLux-STXM as shown in Fig. 10. When using a photon energy of $286.5 \mathrm{eV}$ [Fig. 10(a)], strong absorption from the TCNQ crystallites is observed. The macrocrystal seems to consist of platelike microcrystals that might be rotated with respect to each other. At this photon energy the contrast is related to the sample thickness $t(\vec{r})$, which is approximately uniform as seen from the equal gray level of all crystallites in the image. The rotation of the microcrystallites can be clearly discerned when using a photon energy of $E=284.2 \mathrm{eV}$ [Fig. 10(b)]. Since the light is linearly polarized ( $\vec{P}=$ linear horizontal), we predominantly excite electrons into the unoccupied $\pi^{*}$-states. This absorption depends strongly on the carbon double bond orientation as shown in Fig. 10(b). The different gray levels of the microcrystals indicate the different orientations of their $\mathrm{C}-\mathrm{C}$ double bonds in the constituting molecules. Local spectra acquired from microcrystallites of different orientations are shown in Fig. 10(c). These local spectra confirm that the absorption is independent of crystallite orientation at $E=286.5 \mathrm{eV}$, while absorption differs clearly for differently oriented crystallites at $E=284.2 \mathrm{eV}$. 

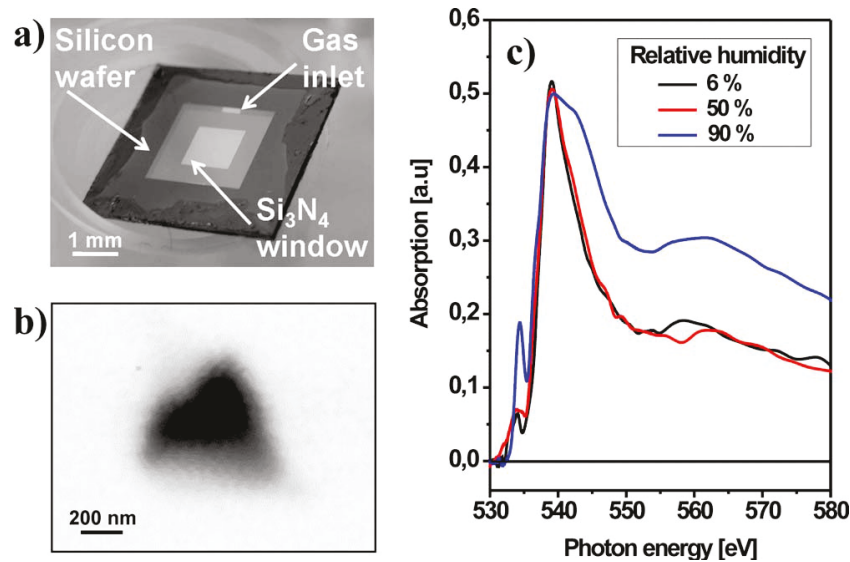

FIG. 11. (Color online) Climate cell (a) consisting of two silicon wafers with $\mathrm{Si}_{3} \mathrm{~N}_{4}$ windows $(t=100 \mathrm{~nm})$ and a gas inlet and outlet (not visible). STXM transmission image taken at $540 \mathrm{eV}$ of an individual particle composed of ammonium sulfate and adipic acid is shown in (b). The dependence of the oxygen $K$-edge spectrum of an ammonium sulfate/adipic acid film on the humidity is show in (c). See text for details.

According to simulations performed by Schöll, ${ }^{48}$ the resonances denoted as A, B, C, and D in Fig. 10(c) can be attributed to the following $\mathrm{C} 1 s \rightarrow \pi^{*}$ excitations: A: cyclohexadiene carbon bonds (C4); B: carbene $(\mathrm{C} 2)$ and substituted cyclohexadiene carbon (C3) bonds; $\mathrm{C}$ : cyclohexadiene carbon (C4) and cyano group bonds (C1); D: cyano group bond (C1) and substituted cyclohexadiene carbon (C3) bonds. Accordingly, one can unambiguously conclude that the molecular plane of the TCNQ molecules in the dark areas of the STXM imaged at $284.2 \mathrm{eV}$ are oriented upright with respect to the silver substrate, whereas in the bright areas molecules lie mostly flat on the substrate. Thus, STXM experiments with polarized lights allow the investigation of organic crystallites with a resolution of several tens of nanometers in addition to identifying not only the size and size distribution of the microcrystallites but also their absolute molecular orientation.

\section{Artificia aerosols under controlled gas phase}

To study samples with a high vapor pressure under a controlled gas atmosphere, a gas-tight climate cell has been developed. The cell is made from a sandwich of two silicon wafers, each $100 \mu \mathrm{m}$ thick [Fig. 11(a)]. Both wafers have a silicon nitride window of $100 \mathrm{~nm}$ thickness in their center. The sample is deposited on the front wafer while the back wafer closes the cell. The custom made wafer at the back of the cell has two additional holes besides the main window, which serve as inlet and outlet for the gas flow. The sample space between the two wafers is sealed air tight using a film of $25 \mu \mathrm{m}$ thick glue (Scotch ${ }^{\mathrm{TM}} 3 \mathrm{~m}$ Art. No. 9458). To secure complete tightness, the outside of the cell was sealed using rubber cement. The cell is mounted on a standard sample plate for the microscope, which was modified to allow connection of the gas supply to the cell. To minimize the $\mathrm{x}$-ray absorption by the gas inside the microscope and the cell, helium is used as buffer gas (pressure $\sim 1$ atm). The gas flow is controlled by mass flow controllers and was chosen as low as conveniently possible (a few $\mathrm{ml} / \mathrm{min}$ ) to avoid any
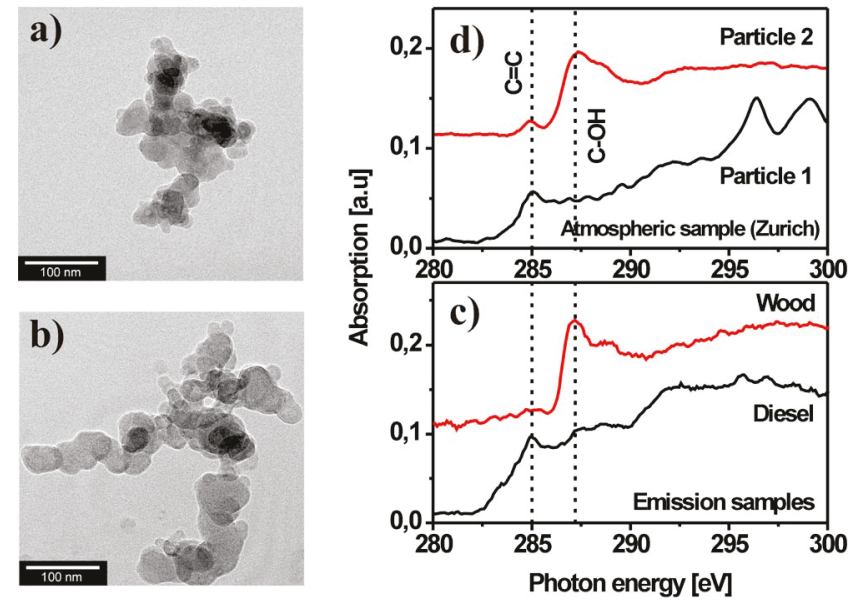

FIG. 12. (Color online) TEM bright field micrograph of (a) soot particle from wood combustion and (b) diesel soot. Both consist of chains of primary particles. C $K$-edge NEXAFS spectra of (c) single soot particles from wood and diesel combustion and (d) from two particles in an atmospheric sample, collected along an arterial road in Zurich.

movement of the sample or bending of the thin silicon nitride membranes. Prior to entering the cell, the gas flow passes over ice or a water film of variable temperature, thereby controlling the humidity.

As proof of concept, we have studied mixtures of an inorganic salt (ammonium sulfate) with an organic acid (adipic acid). Two types of samples have been prepared. First, an aqueous solution of both substances was nebulized; the aerosol was then dried and deposited on the upstream silicon nitride membrane. A typical STXM transmission image of an aerosol particle (size approximately $500 \mathrm{~nm}$ ) recorded at 540 $\mathrm{eV}$ is shown in Fig. 11(b). Second, an aqueous solution of ammonium sulfate and adipic acid was brought onto a membrane, dried and mounted to such a cell. In Fig. 11(c), we demonstrate the response of the $\mathrm{O} K$-edge NEXAFS spectra to a change in humidity in the cell. For a humidity well below the deliquescence humidity of ammonium nitrate ( $80 \%$ relative humidity), the spectrum remains unchanged. Once the relative humidity is above the deliquescence point, the spectral changes reflect both the uptake of water and the phase transition from the solid ammonium sulfate into an aqueous ammonium sulfate solution.

\section{Source speciation of aerosol particles in ambient samples}

The morphology of diesel and wood smoke soot particles is very similar. They are generally smaller than $400 \mathrm{~nm}$ and consist of chains of primary particles with a graphitic structure [see Figs. 12(a) and 12(b)]. Diesel and wood combustion emission samples can, however, be distinguished based on the known C $K$-edge NEXAFS of bulk material. ${ }^{34}$ Characteristic resonances in the spectra allow for direct molecular speciation of the graphitelike solid core, surface functional groups, and aromatic and aliphatic components depending on the origin of the soot. With STXM, it is possible to gain more detailed information on the variation in chemical structure of single soot particles. ${ }^{49}$ We were able to obtain clear spectra of particles as small as $100 \mathrm{~nm}$ by averaging 
data of line scans with $20 \mathrm{~nm}$ step size and $10 \mathrm{~ms}$ dwell time. Diesel soot particles [Fig. 12(c)] contain a dominant spectral signature at $285 \mathrm{eV}$ stemming from unsaturated (multiple) carbon bonds and a shoulder in the spectrum at $\sim 291 \mathrm{eV}$ from equivalent $\mathrm{C}=\mathrm{C} \sigma$-bonds from the graphitic core. The spectra from graphite also show these features, confirming the partial graphitic nature of diesel soot. The spectra of wood soot have a less graphitic nature but show a peak at $287.2 \mathrm{eV}$ from $\mathrm{C}-\mathrm{OH}$ bonds [see Fig. 12(c)]. These source specific signatures allowed discrimination between wood and diesel soot particles in an ambient air sample collected next to an arterial road in Zurich [Fig. 12(d)]. The spectrum of particle 1 shows the characteristics of a diesel combustion source and the spectrum of particle 2 that of wood combustion. Additional peaks are found, for example, between 295 and $300 \mathrm{eV}$ in particle 1. However, the spectra obtained from the atmospheric particles do not completely match the diesel and wood soot spectra. Most probably this is due to the particle aging, i.e., reaction with atmospheric gases and adsorption of new species. In order to investigate this question, the effects of particle weathering by ozone are being conducted at present.

\section{E. Examination of gas-fille microballoons in water environment}

Gas-encapsulated microballoons are of interest as contrast enhancers for ultrasound diagnostics and/or as carriers of therapeutic gases. ${ }^{50,51}$ Very recently, the PolLux STXM has been utilized for the first spectromicroscopy examination of gas-filled microballoons in aqueous solution. ${ }^{52}$ Because the water in which the microballoons are suspended only weakly absorbs x-rays in the photon energy range between 280 and $520 \mathrm{eV}$, x-ray microspectroscopy of such objects in their natural water environment is very useful and an important tool for the development of applications.

In the present example, stable air-filled poly(vinyl alcohol) (PVA)-coated microballoons were prepared by crosslinking telechelic PVA at the water/air interface. ${ }^{53}$ STXM measurements were performed using a wet cell containing approximately $1 \mu \mathrm{l}$ of a water microballoon suspension sandwiched between two $\mathrm{Si}_{3} \mathrm{~N}_{4}$ membranes of $100 \mathrm{~nm}$ thickness, ${ }^{27}$ sealed with high-vacuum grease to maintain the water environment during STXM investigation. Figure 13 shows transmission images $\left(20 \times 20 \mu \mathrm{m}^{2}\right)$ of microballoons in a water environment recorded at (a) $520 \mathrm{eV}$ and (b) 550 eV. The absorption contrast in Fig. 13(a), below the oxygen $K$ edge $(520 \mathrm{eV})$, is due to the strong absorption of the x-rays by the carbonaceous material, whereas the water and air have only weak absorption at this photon energy. Above the oxygen $K$ edge $(550 \mathrm{eV})$, the transmission changes drastically. The inside of the microballoons stays transparent (white), while the surrounding water turns absorbing (dark). This is due to the high oxygen $1 s$ absorption in water and the low absorption in air, primarily due to their density differences. As one can see, the image shows some variations between the particles, namely, differences in the polymeric shell thickness and different absorptions from the internal part of the microballoons. Moreover, the microballoon labeled B shows almost the same absorption signal as the surrounding
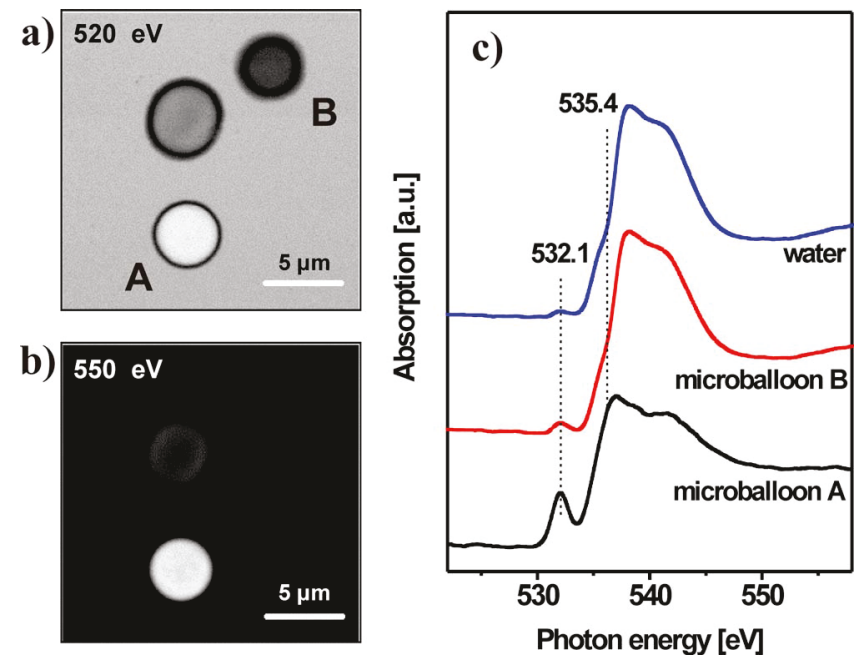

FIG. 13. (Color online) STXM transmission images of microballoons in water environment recorded at (a) $520 \mathrm{eV}$ and (b) $550 \mathrm{eV}$. (c) Oxygen $K$-edge NEXAFS spectra of the surrounding water and the interior parts of microballoons A and B.

water at $550 \mathrm{eV}$ [see Fig. 13(b)]. This suggests that microballoon B is filled with water, a finding that is corroborated by the oxygen $K$-edge NEXAFS spectra. The absorption spectra extracted from line scans across the inner part of particles A and $\mathrm{B}$ are compared in Fig. 13(c). A NEXAFS spectrum acquired from the surrounding water is also shown in this figure. The spectrum from microballoon B shows essentially the same resonances as the water spectrum except a small intensity decrease in the feature at $535.4 \mathrm{eV}$ and an intensity increase in the peak at $532.1 \mathrm{eV}$. This result explicitly suggests the presence of water inside the balloon. In contrast to the spectra of the surrounding water and to particle $\mathrm{B}$, the NEXAFS spectrum of microballoon A shows a strong resonance at $532.1 \mathrm{eV}$, which is assigned to the $\mathrm{O} 1 s \rightarrow \pi_{(\mathrm{C}=\mathrm{O})}^{*}$ transition originating from the carbonyl groups of the telechelic PVA shell. ${ }^{54}$ Furthermore, the water peak at $535.4 \mathrm{eV}$ is absent in this spectrum while the main $\mathrm{O} 1 s \rightarrow \sigma^{*}$ resonance appears at around $537 \mathrm{eV}$. Hence, the NEXAFS spectrum of microballoon A shows only the resonances typical for the telechelic PVA shell. This result strongly suggests that microballoon A is air filled. ${ }^{52}$

\section{SUMMARY}

The new PolLux facility at the Swiss Light Source allows soft x-ray microspectroscopic experiments on a wide range of materials with both high spatial and spectral resolutions. By using two gratings in a spherical grating monochromator an extended energy range from 200 to $1400 \mathrm{eV}$ is covered. The obtainable spectral resolution is at least $E / \Delta E=5000$ at $400 \mathrm{eV}$. Circularly polarized soft $\mathrm{x}$-rays can be obtained by inducing a controlled vertical tilt of the electron beam in the dipole magnet. This tilt is fully compensated within the sector of the electron storage ring and invisible to other beamlines. The PolLux instrument uses interferometric control of the relative position between the FZP and the sample to maintain complete registry of the $x, y$ coordinates as a function of photon energy. Its imaging per- 
formance is evidenced by the smallest structures resolved which are test patterns with $15 \mathrm{~nm}$ lines followed by $15 \mathrm{~nm}$ trenches. Because of its flexible sample environment, which includes heating, cooling, gas and liquid cells, and electrical connections to the outside, the PolLux-STXM offers a very wide variety of experiments from hard- and soft-condensed matter as well as in environmental science and biology.

\section{ACKNOWLEDGMENTS}

We acknowledge financial support through the BMBF (Project No. 05KS4WE1) and BaCaTec. We like to thank Dr. S. Sjoegren and Dr. E. Weingartner for helpful discussion concerning the preparation and the thermodynamics of ammonium sulfate and adipic acid mixtures. The assistance of Dr. M. Ammann and M. Birrer in preparing the climate cell was gratefully acknowledged. We also acknowledge the constructive interaction with Dr. U. Wiesemann and W. Diete from ACCEL. The measurements have been performed at the Swiss Light Source, Paul Scherrer Institut, Villigen, Switzerland.

${ }^{1}$ J. Stöhr, NEXAFS Spectroscopy (Springer, New York, 1992).

${ }^{2}$ H. Ade and A. P. Hitchcock, Polymer 49, 643 (2008).

${ }^{3}$ B. L. Henke, E. M. Gullikson, and J. C. Davis, At. Data Nucl. Data Tables 54, 181 (1993).

${ }^{4}$ J. Kirz and H. Rarback, Rev. Sci. Instrum. 56, 1 (1985).

${ }^{5}$ A. L. D. Kilcoyne, T. Tyliszczak, W. F. Steele, S. Fakra, P. Hitchcock, K. Franck, E. Anderson, B. Harteneck, E. G. Rightor, G. E. Mitchell, A. P. Hitchcock, L. Yang, T. Warwick, and H. Ade, J. Synchrotron Radiat. 10, 125 (2003).

${ }^{6}$ U. Wiesemann, J. Thieme, P. Guttmann, B. Niemann, D. Rudolph, and G. Schmahl, AIP Conf. Proc. 507, 430 (2000).

${ }^{7}$ T. Beetz, M. Feser, H. Fleckenstein, B. Hornberger, C. Jacobsen, J. Kirz, M. Lerotic, E. Lima, M. Lu, D. Sayre, D. Shapiro, A. Stein, D. Tennant, and S. Wirick, Synchrotron Radiat. News 16, 11 (2003).

${ }^{8}$ B. Kaulich, D. Bacescu, D. Cocco, J. Susini, M. Salomé, O. Dhez, C. David, T. Weitkamp, E. D. Fabrizio, S. Cabrini, G. Morrison, P. Charalambous, J. Thieme, T. Wilhein, J. Kovac, M. Podnar, and M. Kiskinova, J. Phys. IV 104, 103 (2003).

${ }^{9}$ K. V. Kaznatcheev, Ch. Karunakaran, U. D. Lanke, S. G. Urquhart, M. Obst, and A. P. Hitchcock, Nucl. Instrum. Methods Phys. Res. A 582, 96 (2007).

${ }^{10}$ H.-J. Shin and M. K. Lee, Nucl. Instrum. Methods Phys. Res. A 467, 909 (2001).

${ }^{11}$ J. M. Kenney, G. R. Morrison, M. T. Browne, C. J. Buckley, R. E. Burge, R. C. Cave, P. S. Charalambous, P. J. Duke, A. R. Hare, C. P. B. Hills, A. G. Michette, K. Ogawa, and A. M. Rogoyski, J. Phys. E 22, 234 (1989).

${ }^{12}$ G. Schneider, Ultramicroscopy 75, 85 (1998); P. Guttmann, B. Niemann, J. Thieme, D. Hambach, G. Schneider, U. Wiesemann, D. Rudolph, and G. Schmahl, Nucl. Instrum. Methods Phys. Res. A 467, 849 (2001).

${ }^{13}$ G. Denbeaux, E. Anderson, W. Chao, T. Eimüller, L. Johnson, M. Köhler, C. Larabell, M. Legros, P. Fischer, A. Pearson, G. Schütz, D. Yager, and D. Attwood, Nucl. Instrum. Methods Phys. Res. A 467, 841 (2001).

${ }^{14}$ G. Schneider, P. Guttmann, S. Heim, S. Rehbein, D. Eichert, and B. Niemann, AIP Conf. Proc. 879, 1291 (2007).

${ }^{15}$ J. H. Dunn, A. Hahlin, O. Karis, D. Arvanitis, G. LeBlanc, E. Andersson, and L.-J. Lindgren, AIP Conf. Proc. 705, 65 (2004).

${ }^{16}$ T. Warwick, H. A. Padmore, and H. Ade, Proc. SPIE 3449, 12 (1998).

${ }^{17}$ A. Lüdeke, A. Andersson, M. Böge, B. Kalantari, B. Keil, M. Pedrozzi, T. Schilcher, V. Schlott, and A. Streun, Proceedings of EPAC, Edinburgh, Scotland, 2006 (unpublished), p. 3424.

${ }^{18}$ U. Flechsig, C. Quitmann, J. Raabe, M. Böge, R. Fink, and H. Ade, AIP Conf. Proc. 879, 505 (2007).

${ }^{19}$ T. Warwick, H. Ade, D. Kilcoyne, M. Kritscher, T. Tyliszczak, S. Fakra, A. Hitchcock, P. Hitchcock, and H. Padmore, J. Synchrotron Radiat. 9, 254 (2002)

${ }^{20}$ C. J. Buckley, in X-Ray Microscopy II, edited by D. Sayre, M. Howells, J.
Kirz, and H. Rarback (Springer, Berlin, 1988), pp. 146-150.

${ }^{21}$ B. Winn, H. Ade, C. Buckley, M. Feser, M. Howells, S. Hulbert, C. Jacobsen, K. Kaznacheyev, J. Kirz, A. Osanna, J. Maser, I. McNulty, J. Miao, T. Oversluizen, S. Spector, B. Sullivan, Y. Wang, S. Wirick, and H. Zhang, J. Synchrotron Radiat. 7, 395 (2000).

${ }^{22}$ S. Henein, U. Frommherz, R. Betemps, H. Kalt, U. Ellenberger, U. Flechsig, and J. Raabe, AIP Conf. Proc. 879, 643 (2007).

${ }^{23}$ Y. Ma, C. T. Chen, G. Meigs, K. Randall, and F. Sette, Phys. Rev. A 44, 1848 (1991).

${ }^{24}$ To keep the spectral resolution $\Delta E / E \sim 2000$ constant, the horizontal width of the entrance slit (S1) was varied: 300 lines/mm grating: S1 $=112 \mu \mathrm{m}(150 \mathrm{eV})$ reduced to $50 \mu \mathrm{m}(600 \mathrm{eV}) ; 600$ lines $/ \mathrm{mm}$ grating: $140 \mu \mathrm{m}(400 \mathrm{eV})$ reduced to $25 \mu \mathrm{m}(1300 \mathrm{eV})$. The vertical size was kept constant. The exit slit (S2) was kept constant at $40 \times 40 \mu \mathrm{m}^{2}$, resulting in a constant spot diameter on the sample. We used a noncalibrated $\mathrm{Si}$ diode and calculated the photon flux from the measured current using the efficiency curve provided by the vendor.

${ }^{25}$ M. Böge, U. Flechsig, J. Raabe, and T. Schilcher, EPAC European Particle Accelerator Conference, Edingburgh, UK, 2006 (unpublished), p. 3610.

${ }^{26}$ ACCEL Instruments GmbH, Bergisch Gladbach, Germany ( http:// www.accel.de/).

${ }^{27}$ Silson Ltd., Northampton, England (http://www.silson.com).

${ }^{28}$ Agilent Technologies (http://www.home.agilent.com/).

${ }^{29}$ K. Jefimovs, J. Vila-Comamala, T. Pilvi, J. Raabe, M. Ritala, and C. David, Phys. Rev. Lett. 99, 264801 (2007).

${ }^{30}$ V. Schlott, R. Kramert, M. Rohrer, A. Streun, P. Wiegand, S. Zelenika, R. Ruland, and E. Meier, Proceedings of EPAC, Vienna, Austria, 2000 (unpublished), p. 693.

${ }^{31}$ H. Ade, X. Zhang, S. Cameron, C. Costello, J. Kirz, and S. Williams, Science 258, 972 (1992).

${ }^{32}$ B. Van Waeyenberge, A. Puzic, H. Stoll, K. W. Chou, T. Tyliszczak, R. Hertel, M. Fähnle, H. Bruckl, K. Rott, G. Reiss, I. Neudecker, D. Weiss, C. H. Back, and G. Schütz, Nature (London) 444, 461 (2006).

${ }^{33}$ Y. Acremann, J. P. Strachan, V. Chembrolu, S. D. Andrews, T. Tyliszczak, J. A. Katine, M. J. Carey, B. M. Clemens, H. C. Siegmann, and J. Stöhr, Phys. Rev. Lett. 96, 217202 (2006).

${ }^{34}$ A. Braun, J. Environ. Monit. 7, 1059 (2005).

${ }^{35}$ S. Bernard, K. Benzerara, O. Beyssac, N. Menguy, F. Guyot, G. E. Brown, Jr., and B. Goffé, Earth Planet. Sci. Lett. 262, 257 (2007).

${ }^{36}$ J. Kirz, C. Jacobsen, and M. Howells, Q. Rev. Biophys. 28, 33 (1995).

${ }^{37} \mathrm{O}$. Dhez, H. Ade, and S. Urquhart, J. Electron Spectrosc. Relat. Phenom. 128, 85 (2003).

${ }^{38}$ J. Stöhr, M. G. Samant, Y. Wu, B. D. Hermsmeier, G. R. Harp, S. Koranda, D. Dunham, and B. P. Tonner, Science 259, 658 (1993).

${ }^{39}$ E. E. Fullerton, O. Hellwig, Y. Ikeda, B. Lengsfield, K. Takano, and J. B. Kortright, IEEE Trans. Magn. 38, 1693 (2002).

${ }^{40}$ S. G. Urquhart, A. P. Hitchcock, A. Smith, H. W. Ade, W. Lidy, E. G. Rightor, and G. E. Mitchell, J. Electron Spectrosc. Relat. Phenom. 100, 119 (1999).

${ }^{41}$ A. Hitchcock, J. Synchrotron Radiat. 8, 66 (2001).

${ }^{42}$ M. Schumacher, I. Christl, A. Scheinost, C. Jacobsen, and R. Kretzschmar, Environ. Sci. Technol. 39, 9094 (2005).

${ }^{43}$ U. Neuhäusler, S. Abend, G. Lagaly, and C. Jacobsen, Colloid Polym. Sci. 277, 719 (1999).

${ }^{44}$ G. E. Mitchell, L. R. Wilson, M. T. Dineen, S. G. Urquhart, F. Hayes, E. G. Rightor, A. P. Hitchcock, and H. Ade, Macromolecules 35, 1336 (2002).

${ }^{45}$ M. Lerotic, C. Jacobsen, T. Schäfer, and S. Vogt, Ultramicroscopy 100, 35 (2004).

${ }^{46}$ S. G. Urquhart and H. Ade, J. Phys. Chem. B 106, 8531 (2002).

${ }^{47}$ H. Ade and B. Hsiao, Science 262, 1427 (1993).

${ }^{48}$ A. Schöll, Ph.D. thesis, Julius-Maximilians-Universität Würzburg, 2003.

${ }^{49}$ R. J. Hopkins, A. V. Tivanski, B. D. Marten, and M. K. Gilles, J. Aerosol Sci. 38, 573 (2007).

${ }^{50}$ P. A. Dayton and K. Ferrara, J. Magn. Reson Imaging 16, 362 (2002).

${ }^{51}$ J. R. Lindner, Nat. Rev. Drug Discovery 3, 527 (2004).

${ }^{52}$ G. Tzvetkov, B. Graf, P. Fernandes, A. Fery, F. Cavalieri, G. Paradossi, and R. H. Fink, Soft Matter 4, 510 (2008).

${ }^{53}$ F. Cavalieri, A. El Hamassi, E. Chiessi, and G. Paradossi, Langmuir 21, 8758 (2005).

${ }^{54}$ K. C. Prince, R. Richter, M. de Simone, M. Alagia, and M. Coreno, J. Phys. Chem. A 107, 1955 (2003). 